\title{
Grupos vulnerables y uso del espacio público en el centro histórico de San Salvador. Caso calle Arce
}

\author{
Vulnerable groups and the use of public space in historic downtown \\ San Salvador. The case of Calle Arce
}

\section{ISSN 2071-8748 \\ E-ISSN 2218-3345 \\ (c) $($ ()}

URI: http://hdl.handle.net/11298/1123

DOI: https://doi.org/10.5377/entorno.v0i68.8443
Melissa Regina Campos-Solórzano Universidad Tecnológica de El Salvador melissa.campos@utec.edu.sv ORCID: 0000-0002-8436-0809

Recibido: 4 de junio de 2019 Aprobado: 5 de septiembre de 2019

\section{Resumen}

Este artículo resume los resultados de una investigación cualitativa sobre los usos que las personas, particularmente pertenecientes a algunos grupos vulnerables, hacen de la calle Arce como espacio público de la capital salvadoreña, con el fin de producir mediante el análisis y conjunción de la información recabada, una síntesis, de cara a los recientes proyectos de intervención física en la ciudad, que contribuya al entendimiento holístico del territorio para generar acciones tendientes la re-apropiación integral del espacio. Se realizaron observaciones y entrevistas con algunas personas dispuestas a colaborar. La reflexión final es que la Calle Arce, a pesar de ser un espacio público por definición, en la práctica -pese a los

\section{Abstract}

This article summarizes the results of a qualitative research conducted on the use that people make of Calle Arce as part of the public space in the salvadorean capital city, especially by those ones who belong to vulnerable groups. The purpose is to synthesize the current situation by means of the analysis and conjunction of the gathered information, in the face of the recent projects, to physically intervene the city in order to contribute to the holistic understanding of the territory with the purpose of generating actions aimed at the re-appropriation of space in an integral manner. Field observations and interviews with people interested in collaborating were conducted. The final 
esfuerzos municipales- no es un espacio inclusivo debido la apropiación y lucha constante de espacios en la vía.

\section{Palabras clave}

Rehabilitación urbana - Calle Arce - San Salvador, El Salvador. Urbanismo - Calle Arce - San Salvador, El Salvador. Desarrollo urbano - Calle Arce - San Salvador, El Salvador reflection is that Calle Arce, in spite of being a public space by definition, in practice-notwithstanding the municipal efforts-- is not an inclusive space because of the appropriation and constant struggle for space on that street.

\section{Keywords}

Urban rehabilitation - Calle Arce - San Salvador, El Salvador. Urbanism - Calle Arce - San Salvador, El Salvador. Urban development - Calle Arce - San Salvador, El Salvador

\section{Introducción}

El objetivo central de la investigación fue reconocer los usos que las personas en estado de vulnerabilidad hacen del espacio de la calle Arce, en el centro histórico de San Salvador, El Salvador. Para ello, se establecieron como objetivos específicos identificar los diversos grupos de la población que se encuentran en estado de vulnerabilidad en el corredor de la calle; diferenciar las particularidades del uso entre los diferentes grupos de personas que transitan y habitan la calle; comparar el uso del espacio público entre hombres y mujeres; e interpretar las razones que dan sentido al uso particular del espacio público de parte de los grupos vulnerables.

Al abordar como sujeto de estudio a los grupos vulnerables, nos referimos a aquella población que, por el hecho de transitar o habitar en un determinado espacio urbano, puede verse afectada por la problemática socioeconómica, política y cultural: pandillas, inseguridad, acoso, violencia, irrespeto de conductores, servicios básicos inexistentes, invasión del espacio peatonal, ventas ambulantes, discriminación, estigmatización de la pobreza, entre otros (Gravano, 2016). De tal forma que el término no hace referencia particular a la existencia de una situación crítica, sino a las condiciones de riesgo o desventaja que para algunas personas representa una mayor probabilidad de sufrir esas situaciones de alteración de la cotidianidad (Hernández, 2007). Existen múltiples estudios en Latinoamérica que dan cuenta de cómo el ejercicio de mitigación de las vulnerabilidades genera efectos positivos al acompañar proyectos de intervención urbana; los más representativos se encuentran en Colombia, México y Argentina, por lo que, tomando de ejemplo sus buenas prácticas, El Salvador debe hacer un ejercicio de abordar el estudio de la ciudad como un organismo vivo.

Este artículo es producto de una investigación de carácter cualitativo, cuya premisa resalta el derecho a la ciudad, concepto popularizado por el francés Henri Lefevbre a mediados del siglo pasado, pero que trasciende a la actualidad cuando encontramos sectores de la sociedad al margen de los servicios y consumos colectivos que la ciudad brinda, o debería hacerlo. El derecho a la ciudad debe entenderse entonces como un derecho a la vida urbana, a lugres de encuentro y cambios, a los ritmos de la vida y empleos del tiempo que permiten el uso pleno de estos encuentros y lugares (Hernández y Díaz, 2018).

Los resultados de esta investigación pretenden ser insumo de discusión, y a la vez plantean las principales contrariedades que impiden que se disfrute de la ciudad como derecho. Sin embargo, existe un conflicto importante entre las modalidades socioétnicas de ocupación del espacio urbano en San Salvador, evidente, por ejemplo, en la ocupación de la calle como espacio público por parte de los vendedores ambulantes y la organización moderna del comercio informal; un fenómeno que no es particular de nuestro territorio, sino común en Latinoamérica, tanto que el urbanista francés Jérôme Monnet ha llegado a afirmar que no hay espacio público, más bien hay espacios colectivos de flujo y espacios comunitarios de identificación, lo cual impulsa el replanteamiento que las formas de intercambio reformulan el sentido de lo público en variadas competencias 
con las historias y tradiciones de cada sociedad (García Canclini, 1996).

El espacio público en el centro de la capital, y específicamente en el área de la calle Arce, sufre pérdidas importantes en cuanto a su dimensión de uso y a su valor de paisaje urbano. Las plazas, los parques, las calles y sendas peatonales son invadidos y tomados por los vendedores ambulantes. Las plazas han pasado de ser lugares de encuentro a lugares de intercambio, las aceras y veredas peatonales son sitios de venta de paso, segmentadas, interrumpidas y fraccionadas hasta perder por completo su función y forma de uso. (Granadillo en Menjivar 2009).

Ante el evidente caos, entra en discusión el estudio de la percepción geográfica, cuyo planteamiento teórico admite que el ser humano recoge una serie de imágenes del medio en su mente y que a través de su experiencia construye su propio esquema cognitivo de la realidad espacial, por lo que se plantea la necesidad de analizar la ciudad desde el punto de vista de los sujetos, reconociendo la constante interacción que existe entre el ser humano y su medio, y que concluye con la elaboración de una imagen mental del conjunto urbano. La configuración de este imaginario supone una comprensión del medio, que el urbanista estadounidense Kevin Lynch evaluó como los atributos esenciales del arraigo o la identificación que tienen los habitantes respecto a su entorno (Muñoz y Catalán, 2015).

\section{Metodología}

La base de la investigación se llevó a cabo mediante un enfoque cualitativo de tipo etnográfico básico con unidad de análisis basada en observación y complementado con indagación documental.

Para Hernández, Fernández y Baptista (2014), los estudios cualitativos se enfocan en comprender los fenómenos explorándolos desde la perspectiva de los participantes en un ambiente natural y en relación con su contexto.

Se realizó una intensiva revisión de la literatura con el fin de detectar conceptos clave y entender mejor los resultados, evaluar las categorías relevantes y profundizar en las interpretaciones. El planteamiento se fundamentó en las investigaciones previas, pero fue amoldándose en medio del proceso mismo de inmersión en el contexto, de la recolección de los primeros datos y su análisis.
El ambiente en el que se llevó a cabo el estudio fue el eje de la calle Arce, comprendido, de oriente a poniente, desde el costado oeste de la catedral metropolitana hasta la Plaza de la Salud, entre marzo del 2018 y febrero del 2019.

La muestra seleccionada fue de caso tipo debido a que el objetivo de la investigación radicó en obtener riqueza, profundidad y calidad de la información.

En vista de la poca apertura de parte de los sujetos de estudio, se optó por la aplicación de la observación investigativa densa y profunda para explorar y describir los ambientes, los comportamientos y sus significados e identificar posibles problemas sociales. (Fernández y Baptista, 2014). Posteriormente, y como resultado de la observación, la investigación se enfocó principalmente en las siguientes unidades de análisis: a) entorno, b) ambiente social y humano, c) actividades individuales y colectivas.

Los registros y anotaciones se realizaron posteriormente al trabajo de campo, ya que durante las primeras aproximaciones se notaba incomodidad y actitudes a la defensiva de los sujetos de estudio. Las grabaciones de audio, video y fotografías fueron casi nulas dada la inseguridad de la zona para portar los equipos y/o la falta de aprobación por parte de los entrevistados, quienes lo pensaban más de una vez para conversar con el equipo investigador.

\section{Discusión}

La actual historia urbana de San Salvador comienza en 1545 luego de que fuera trasladada de su anterior ubicación en la hacienda La Bermuda. En este nuevo punto, se trazó el origen de la ciudad capital que hoy conocemos, y fue en las cercanías del río Acelhuate, según Gustavo Herodier (1997)، por requerimientos establecidos en las Ordenanzas reales para fundar ciudades españolas de 1538.

Con el paso del tiempo, y 300 años después, El Salvador se posicionaba como uno de los mayores exportadores de café. Debido a las ganancias que ello le generaba, se construyeron edificaciones con influencias inglesa y francesa importadas por las élites que regían al país; edificaciones que se destruyeron con uno de los peores sismos registrado en 1873.

A finales del siglo XVII, en 1885, se erige la para entonces llamada 7. ${ }^{a}$ calle Poniente, también llamada Calle del Hospital Rosales por el recién construido nosocomio del 
mismo nombre y en el que culminaba la calle; hasta que en 1927 cambió su nombre a calle Arce en honor al prócer y primer presidente de las Provincias Unidas de América Central, Manuel José Arce (Grant, 1999).

La calle Arce, en la actualidad (siglo XXI), abarca un total de 12 cuadras - de este a oeste- en la cual comienza el trazo urbano dentro del centro histórico de San Salvador y, a su vez, conforma el eje que divide a la ciudad en norte y sur. Se trata de un corredor desde el cual puede contarse la historia urbana de San Salvador, mediante la identificación de los sitios de memoria que se encuentran edificados a lo largo de la emblemática calle, que se unen con las historias de los transeúntes y habitantes que usan el espacio, en el que han tenido lugar múltiples manifestaciones socioculturales desde su época de esplendor a comienzos de 1900, pero que ha ido cambiando a través del tiempo debido a las prácticas de las personas hasta convertirse, hace algunos años, en un espacio tomado por vendedores ambulantes y cuyo proceso de resignificación está aún en marcha.

El éxodo hacia el occidente de élites que vivieron en el centro de San Salvador, junto con la tendencia de construir nuevos centros exclusivos, ocasionó que desde 1930 a 1960, con el abandono de las mansiones, estas se convirtieran en mesones o centros de comercio para consumidores finales, llevando a otro tipo de ocupación del espacio. Sucesivamente fueron añadiéndose puestos de comercio informal. En el periodo municipal de 1974-1976, el alcalde José Antonio Morales Erlich autorizó la presencia de vendedores en las calles, en la calle Arce hasta la intersección con la 7.a calle; y desde entonces ese límite fue ampliándose a voluntad de los vendedores informales hasta llegar a la toma de la calle completa.

En diciembre de 2010, el entonces alcalde Norman Quijano inició un decidido proceso de reordenamiento, en tres fases, para reubicar a los vendedores. La primera fase del rescate de la calle Arce consistió en construir la Plaza de la Salud. En la segunda etapa se realizaron obras de mejoramiento desde la 23.a hasta la 19.a avenida Norte. En la tercera fase se liberó de ventas desde la 19.a hasta la 9.a avenida Norte. Asimismo, con fondos la Agencia Andaluza de Cooperación Internacional para el Desarrollo se remozaron las aceras y se incluyeron rampas para dar accesibilidad a personas con discapacidad motriz $(E D H, 2016)$.

Seguidamente, en 2016, durante la administración de Nayib Bukele se inició el proceso de recuperación del centro histórico. En su primera fase, se remodelaron los contornos de la Plaza Morazán, Catedral Metropolitana del Divino Salvador del Mundo, Palacio Nacional, Teatro Nacional y otras zonas aledañas de estos para dignificar la capital. Por ello, los comerciantes informales fueron trasladados temporalmente a las zonas previamente recuperadas del pasaje Montalvo, de la 1.a Avenida, calle Arce y de la 6.a calle Oriente, mientras eran reubicados en el mercado Cuscatlán (que se encontraba en trabajos de adecuación) y en el predio de la ex Biblioteca Nacional (Co-Latino, 2016).

Contrario a lo dicho, la solución temporal de reubicación de vendedores en la calle Arce se volvió permanente, y con ella varios vendedores que fueron removidos durante el periodo del 2009 al 2016 volvieron a ocupar sus antiguos puestos. Con el transcurso del tiempo más vendedores se establecen cada semana en los alrededores de la vía, sumándose a una serie de fenómenos de lo urbano que afecta principalmente a individuos pertenecientes a grupos vulnerables, los cuales a grandes rasgos se resumen en los siguientes puntos.

En cuanto al entorno, es difícil describir el ambiente físico de la calle Arce como un espacio homogéneo debido a la distribución de sitios con funciones centrales: plazas, hospitales, centros de estudio medio y superior, mercados al aire libre, comedores, ventas ambulantes, predicadores, servicios de transporte público y privado. Llama particularmente la atención que la ciudad no se encuentra equipada para atender las necesidades de aseo mínimo para personas en situación de calle, pues, aunque no es frecuente, en ocasiones durante la observación de la presente investigación, se identificaron personas que aprovechan la caída de aguas lluvias para lavarse los dientes o para bañarse ante los ojos de asombro y desagrado de los transeúntes, sin embargo, no existe una ducha pública en todo el centro histórico y los baños sanitarios son escasos para la extensión del territorio. Además, el problema de los desechos sólidos, a consecuencia del mal manejo de la basura, es imperante en la mayoría de esquinas de la calle, donde coincide en espacio con los puestos de ventas emplazados en espacios sobre la vía pública, y donde frecuentemente se encuentran cerca niños que acompañan a los vendedores durante su jornada. Asimismo, las bancas, pensadas originalmente como punto de encuentro 0 descanso para las personas agotadas, en su mayoría se encuentran en estado deplorable o son ocupadas como mostradores de los artículos en venta del sector informal. 
Por otro lado, el ambiente social y humano se define por las formas de organización en colectivos que comparten su condición o propósitos: transeúntes, estudiantes, vendedores formales $y$ sindicatos de vendedores independientes, personas en situación de calle, entre otros. Y es en este apartado donde entra en juego la percepción de quienes frecuentan el centro capitalino y quienes lo desconocen por completo; o la percepción de quienes han sufrido alguna situación dañina de quienes no. Para la percepción de algunos, el centro es un espacio caótico donde solo unos pocos se atreven transitar, para otros es el espacio que inevitablemente deben recorrer para llegar a sus destinos, $y$, por el contrario, otros ven en la calle su casa, su hábitat.

Finalmente, las acciones que llevan a cabo los participantes, ya sea de forma individual o colectiva, son desde el transitar a lo largo de la vía por un fin determinado, la venta y compra de productos de primera necesidad o de temporada a bajo precio y la ocupación de los espacios techados como dormitorio por las personas sin hogar.

\section{Conclusiones}

La Nueva Agenda Urbana (2017) plantea el ideal común de una ciudad para todos, refiriéndose a la igualdad en el uso y el disfrute de las ciudades, buscando promover la inclusividad y garantizar que todos los habitantes, tanto de las generaciones presentes como futuras, sin discriminación de ningún tipo, puedan crear ciudades y asentamientos humanos justos, seguros, sanos, accesibles, asequibles, resilientes y sostenibles y habitar en ellos, a fin de promover la prosperidad y la calidad de vida para todos.

Tomando en consideración que el eje de la calle Arce se encuentra estratégicamente ubicado entre los iniciados proyectos de re-significación de la capital, tal como la remodelación del Parque Cuscatlán y la revitaliación del centro histórico, se consideró pertinente contribuir desde la investigación a la interpretación y determinación de las causas que hacen que el espacio comunitario de la calle Arce sea más o menos usado por grupos vulnerables de la sociedad salvadoreña.

Estudios previos al proyecto de intervención del Parque Cuscatlán evidenciaron que las mujeres eran quienes menos hacían uso del espacio y que, por el contrario, personas en situación de calle habitaban al interior del parque. Por lo que, si se busca avanzar hacia la consecución del Objetivo de Desarrollo Sostenible 11, sobre ciudades, asentamientos humanos inclusivos, seguros, resilientes y sostenibles, debemos trabajar sobre el desarrollo de sus metas, como por ejemplo, proporcionar acceso universal a zonas verdes y espacios públicos seguros, inclusivos y accesibles, en particular para las mujeres y los niños, las personas de edad y las discapacitadas, así como aumentar la urbanización inclusiva y sostenible, y la capacidad para la planificación y la gestión participativas, integradas y sostenibles (UN, 2015)

\section{Referencias}

Elsalvador.com. (30 de junio del 2016). Ventas informales vuelven a la calle Arce en el centro. Elsalvador. com. Recuperado de: https://historico.elsalvador. com/196903/ ventas-informales-vuelven-a-la-callearce-en-el-centro.html

García Canclini, N. (1996). Público-privado: La ciudad desdibujada. Alteridades, 6(11), 5-10. Recuperado de http://www.redalyc.org/articulo.oa?id=7471133900

Granillo, H. (2005). El rescate del espacio, una prioridad. En A. S. Menjívar de Síntigo (Com.), Centro histórico de San Salvador: Nuestro reto pendiente (p. 72). San Salvador. FUNDASAL. Recuperado de http://repo.fundasal.org. SV/195/1/CHSS_RETO\%2OPENDIENTE.pdf

Gravano, A. (2016). Antropología de lo urbano. Santiago, Chile: LOM. https://books.google.com.sv/book $\mathrm{s}$ ? id=S5nuDQAAQBAJ\&printsec $=$ frontcover \&dq =Antropolog\%C $3 \% A D a+d e+l o+$ urbano\&hl=es419\&sa $=$ X\&ved=0ahUKEwi8j-Hw_O_mAhUSwlkKHQ RyBBIQ6AEIJZAA\#v=onepage \&q=Antropolog\%C3\%A Da\%20de\%20lo\%20urbano\& $f=$ false

Hernández Aja, A. (julio-agosto, 2007). Áreas vulnerables en el centro de Madrid. Cuadernos de Investigación Urbanística, (53). Recuperado de http://polired.upm. es/index.php/ciur/article/view/268/263

Hernández, J. M. y Díaz García, V. (2018). Visiones del hábitat en América Latina: Participación, autogestión, habitabilidad. Barcelona, España: Editorial Reverté.

Hernández Sampiere, R., Fernández Collado, C. y Baptista Lucio, M. P. (2014). Metodología de la investigación (6 ${ }^{a}$ ed.) [versión de Adobe Acrobat Reader]. Recuperado de http://observatorio.epacartagena.gov.co/wp-content/ uploads/2017/ 08/metodologia-de-la-investigacionsexta-edicion.compressed.pdf

Herodier, G. (1997). San Salvador: El esplendor de una ciudad 1880-1930. San Salvador, El Salvador: ASESUIZA. 
Martínez, D. (20 de abril del 2016). Alcaldía de San Salvador inicia recuperación del centro histórico. Diario Co Latino. Recuperado de https://www.diariocolatino. com/alcaldia -de-san-salvador-inicia-recuperacion-delcentro-historico/

Muñoz Villalobos, D. y Catalán Neumann, R. (diciembre, 2015). Percepción urbana: Imagen de la ciudad de Talca según población adolescente. Revista electrónica de geografía austral, 7(2). Recuperado de http://revistanadir.yolasite.com/resources/ PERCEPCI\%C3\%93N\%20URBANA\%20nadir\%20 2015.pdf

Naciones Unidas. (2017). Nueva agenda urbana [versión de Adobe Acrobat Reader]. Recuperado de http:// habitat3.org/wp-content/uploads/NUA-Spanish.pdf Naciones Unidas. (s. f.). Objetivos de desarrollo sostenible. Recuperado de https://www.un. org/ sustainabledevelopment/es/cities/ 